PACS 61.10.Nz, 61.72.Tt, 79.60.Jv, 78.55.-m

\title{
Formation of silicon nanoclusters in buried ultra-thin oxide layers
}

\author{
O.S. Oberemok*, V.G. Litovchenko, D.V. Gamov, V.G. Popov, V.P. Melnik, \\ O.Yo. Gudymenko, V.A. Nikirin, I.M. Khatsevich \\ V. Lashkaryov Institute of Semiconductor Physics, NAS of Ukraine, \\ 41, prospect Nauky, 03028 Kyiv, Ukraine \\ *Phone/fax: +(38044)-525-5724, e-mail ober@isp.kiev.ua
}

\begin{abstract}
The peculiarities of buried layer formation obtained by co-implantation of $\mathrm{O}_{2}$ ions with the energy of $130 \mathrm{keV}$ and carbon ions within the energy range of 30-50 keV have been investigated. The corresponding ion doses for carbon and oxygen ions were equal to $2 \times 10^{16} \mathrm{~cm}^{-2}$ and $1.8 \times 10^{17} \mathrm{~cm}^{-2}$, respectively. It has been observed that annealing at $1150{ }^{\circ} \mathrm{C}$ results in enhanced oxygen diffusion towards the region with a maximum carbon concentration. Analysis of X-ray diffraction patterns with a SIMS depth profiles inherent to annealed samples suggests formation of Si nanoclusters in the region with maximum concentrations of carbon and oxygen vacancies. The intensive luminescence has been observed with the maximum at $600 \mathrm{~nm}$, which could be associated with silicon nano-inclusions in thin stoichiometric $\mathrm{SiO}_{2}$ layer.
\end{abstract}

Keywords: silicon, nanocluster, buried layer, ion implantation, defects.

Manuscript received 24.11.10; accepted for publication 14.09.11; published online 21.09.11.

\section{Introduction}

Silicon nanocrystalline particles $(\mathrm{nc}-\mathrm{Si})$ are actively studied since there are some prospects of their application for micro- and optoelectronic devices (lightemitting devices, memory cells, etc.) [1-3]. For silicon nanocrystal synthesis, annealing of non-stoichiometric silicon oxide $\left(\mathrm{SiO}_{\mathrm{x}}\right)$ films [4] or $\mathrm{SiO}_{2}-\mathrm{Si}$ structures implanted with silicon ions $[1-3,5]$ are used. When the buried $\mathrm{SiO}_{2}$ film is formed using SIMOX (silicon implanted by oxygen) technology, it has been shown that the films contain silicon inclusions [6].

As a rule, the dimensions of such inclusions lie within the range of 10 to $100 \mathrm{~nm}$, and using those for light emission is impossible. At the same time, synthesis of the buried insulating layers containing silicon nanocrystals has some prospects of practical application in multilayer optoelectronic circuits.

The new SIMOX technology providing stimulated formation of ultra-thin $(\sim 60 \mathrm{~nm}) \mathrm{SiO}_{2}$ layer buried at various depths under the surface of silicon wafer was demonstrated in [7]. $\mathrm{SiO}_{2}$ layer formation was enhanced by carbon implantation inside the buried oxygenenriched layer and subsequent annealing at various temperatures.
In this work, the influence of additional carbon implantation on silicon nanocluster formation in a buried oxide (BOX) layer was investigated using the set of experimental methods.

\section{Materials and methods}

All the silicon samples (n-type, 100 orientation) were implanted with $\mathrm{O}_{2}{ }^{+}$ions (energy $130 \mathrm{keV}$, dose $\left.1.8 \times 10^{17} \mathrm{~cm}^{-2}\right)$. A part of the samples was implanted with carbon ions possessing the energies $30 \mathrm{keV}$ (dose $2 \times 10^{16} \mathrm{~cm}^{-2}$ ) or $50 \mathrm{keV}\left(\right.$ dose $2 \times 10^{16} \mathrm{~cm}^{-2}$ ). Furnace annealing was carried out at $1150{ }^{\circ} \mathrm{C}$ in argon atmosphere for $20 \mathrm{~min}$.

Our investigations of peculiarities of defect creation and transformation were carried out using the X-ray diffuse scattering (XDS) technique with the highresolution diffractometer PANalytical X'Pert Pro MRD.

The XDS intensity distribution $I(\mathbf{q})$ was measured in the vicinity of the 004 node. The $\mathbf{q}$ vector components in the diffraction plane are related with the experimental conditions by the following expression:

$\left(q_{z}, q_{x}\right)=\left(\eta \cos \vartheta_{\mathrm{B}}(2 \Delta \alpha-\Delta \eta) \sin \vartheta_{\mathrm{B}}\right) / \lambda$ 
where $\lambda$ is the X-ray wavelength, $\eta$ and $\Delta \alpha-$ angle deviations of the analyzer and monochromator, correspondingly, $\vartheta_{\mathrm{B}}-$ Bragg angle, $z$ axis is directed normally to the surface, and $x$ axis is parallel to the sample surface; these angles are related within the diffraction plane.

The method to determine the micro-defect (MD) concentration consists in finding the Huang and thermodiffuse scattering ratio, excepting influence of the scattering layer thickness, structure amplitude, spatial angle of scattering, and Debye-Waller factor. So, such important parameters as the micro-defect symmetry, dimension, and concentration may be directly determined from experimental data according to diffuse scattering observed in the investigated sample.

Secondary ion mass spectrometry (SIMS) was used to investigate the dopant distribution along the sample depth. The SIMS measurements have been performed using the Cameca IMS 3F (France) instrument with a $10 \mathrm{keV}$ energy and a $0.4 \mu \mathrm{A}$ current of the primary argon (Ar) ion beam. The sample sputtering area has a rectangular shape with the sizes of $250 \times 250 \mu \mathrm{m}$. The analyzed area was $150 \mu \mathrm{m}$ in diameter at the center of the sputtering area. The sputtering rate was approximately $0.5 \mathrm{~nm} / \mathrm{s}$. The depth scale was determined for each profile by measuring the crater depth with a Dektak 3030 stylus profilometer (Veeco Instruments, USA). Photoluminescence (PL) spectra were measured after plasma ion etching the top Si layer with excitation by a heliumcadmium laser. The thickness and position of the BOX layer were investigated using TEM (transmission electron microscopy) in a cross-section mode. The high-voltage TEM Hitachi H-800 instrument was used.

\section{Results and discussion}

Profiles of oxygen atom distribution for the samples after oxygen ion implantation and combined implantation with oxygen and carbon ions after annealing at $T=1150^{\circ} \mathrm{C}$ are shown in Fig. 1 .

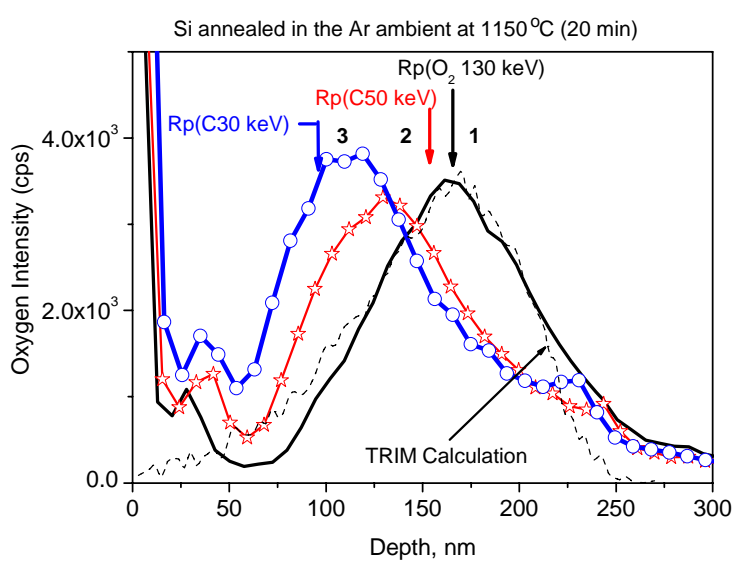

Fig. 1. Profiles of oxygen depth distribution after annealing for the samples implanted with oxygen (1) and additionally implanted with carbon ions possessing the energies $50 \mathrm{keV}$ (2) and $30 \mathrm{keV} \mathrm{(3).}$
After oxygen implantation, the oxygen distribution profile coincides with the calculated (using the TRIM software) one. After additional carbon implantation, the oxygen distribution profile shifts to the sample surface, and is placed within the region between the maximum of carbon distribution at a given energy and maximum of vacancy concentration created in the course of oxygen implantation. For the samples additionally implanted by carbon with the energy $30 \mathrm{keV}$, observed is the region of $\sim 30$-nm depth with a constant oxygen concentration, which is an evidence of thin stoichiometric $\mathrm{SiO}_{2}$ layer creation.

TEM images of the sample cross-sections after oxygen implantation (a), and additional carbon implantation $(b)$ are shown in Fig. 2. One can observe a great amount of defects in the implantation region. The defect region of the samples additionally implanted with carbon is shifted toward the sample surface. For the samples implanted merely by oxygen, zone of interstitial defects behind the implantation region is observed.

The XDS curves for the samples after oxygen implantation (curve 1), carbon co-implantation (curve 2), and after annealing of these samples (curves 3,4) are presented in Fig. 3.
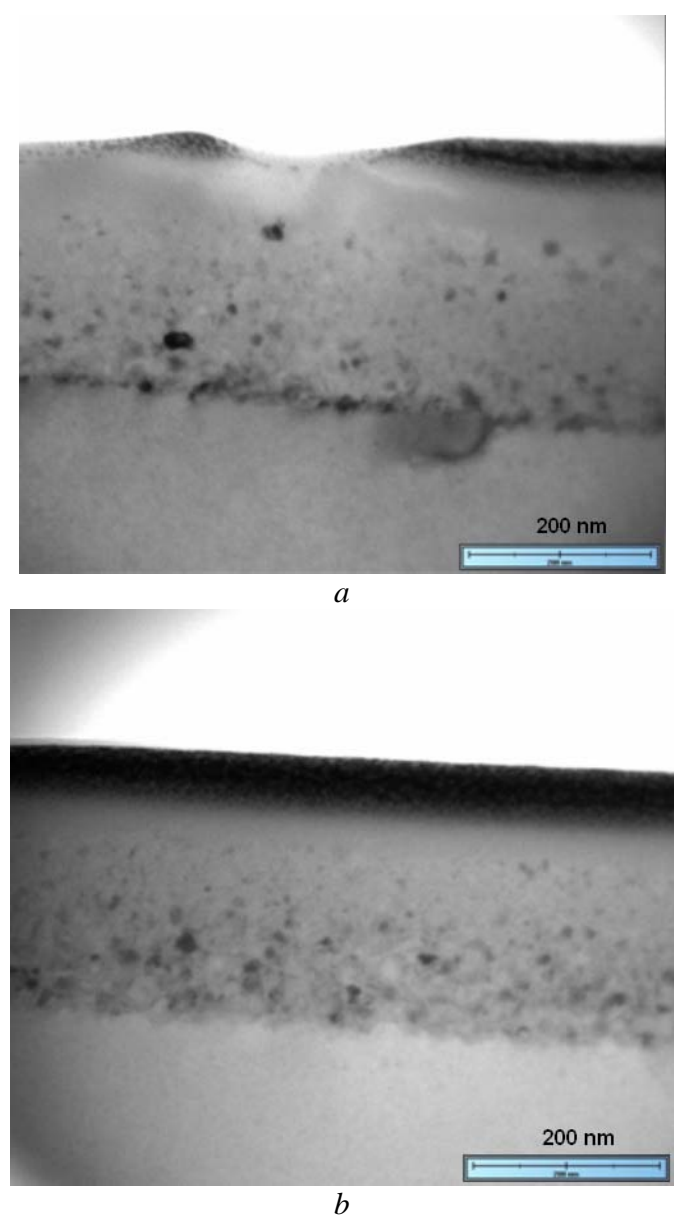

Fig. 2. Cross-section TEM image of the samples implanted with oxygen ions $(a)$ and additionally implanted with carbon ions $(b)$ possessing the energy $30 \mathrm{keV}$, after annealing. 


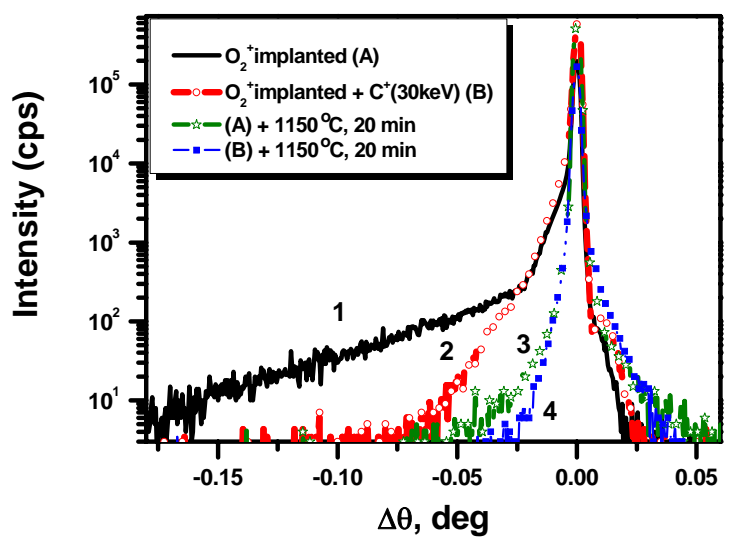

Fig. 3. The XDS curves for the samples after oxygen implantation (curve 1), carbon co-implantation (curve 2), and after annealing of these samples (curves 3,4$) . \Delta \theta=\vartheta-\vartheta_{\mathrm{B}}$.

The half-width of XDS peaks represents the integral characteristic of crystal perfection and is approximately $5.6 \mathrm{arcsec}$. It is very close to the theoretical value and testifies the sample perfection. We have used the diffusion scattering method to obtain the MD parameters. The picture of diffuse scattering distribution in the direction parallel to the reciprocal lattice vector $\left(q_{z}\right.$-section) is shown in Fig. 4, where $\mathbf{q}$ vector from the reciprocal lattice node to the point of measurement for implanted samples before and after annealing.

The $I(\mathbf{q})$ distribution indicates that all the samples have the MD both of vacancy $\left(q_{z}<0\right)$ and interstitial $\left(q_{z}>0\right)$ types. One can see from Fig. 4 that the normalized intensity $I(\mathbf{q}) q^{3}$ is not similar over the whole $q_{z}$ interval. It means that scattering takes place in the asymptotic region, where the defect power $C$ is proportional to $\mathbf{q}>>(\mathbf{Q} C)^{-1 / 2}$. Here $\mathbf{Q}$ is the vector of reciprocal lattice. It is easily to obtain the MD dimensions from the coordinates of the point of transition to horizontal character on the scattering dependence. The samples before annealing are characterized by a high MD concentration $\left(>10^{11} \mathrm{~cm}^{-3}\right.$ ) with the dimensions of $\sim 10$ to $1000 \mathrm{~nm}$. Scattering intensity values for the samples before annealing for the negative $q_{z}$ are substantially higher than those for the positive ones. It means that the total capacity of the vacancy-type MD is higher (approximately 2 times) than that of the interstitial ones.

After ion implantation, substantial diffuse scattering in the region of negative angles is observed in the XDS curves. It points out the high concentration of the vacancy defects. Carbon implantation leads to the substantial scattering decrease in the region of negative $q_{z}$ owing to vacancy trapping of carbon atoms. In this case, the scattering is observed in the region $q_{z}>0$, which is caused by the interstitial component due to additional embedding the interstitial atoms at carbon implantation, and compensation of stretching tensions in the sub-surface region.

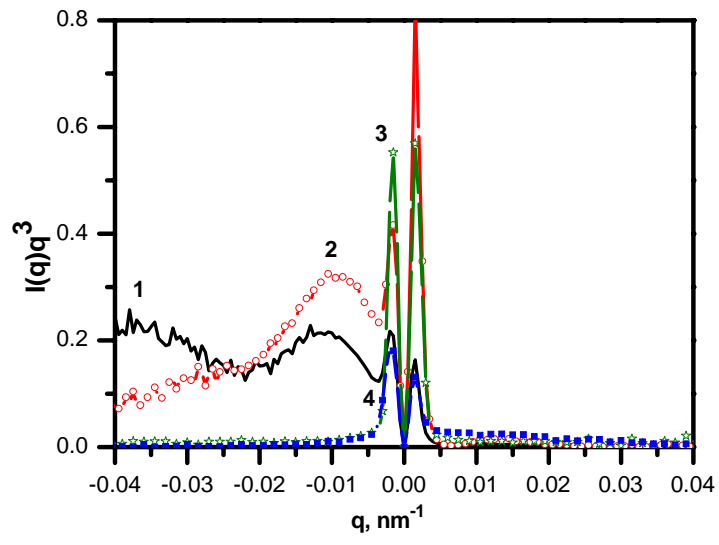

Fig. 4. Dependence of the normalized intensity $I(\mathbf{q}) q^{3}$ on $\mathbf{q}$ vector: for $\mathrm{O}_{2}{ }^{+}$-implanted $\mathrm{Si}$ before (curve 1) and after (curve 3) annealing; for $\mathrm{O}_{2}{ }^{+}$-implanted $\mathrm{Si}$ with additional $\mathrm{C}^{+}(30 \mathrm{keV})$ implantation in the vacancy region before (curve 2) and after (curve 4 ) annealing.

After annealing, a substantial decrease of the diffuse scattering is observed. For the additionally carbon-implanted samples, the scattering component at $q_{z}>0$ is some higher than that for the samples implanted only by oxygen.

During oxygen-implanted sample annealing, the $\mathrm{SiO}_{2}$ phase is formed. Excess silicon atoms recombine with vacancies in the region closer to the surface, also interstitial defect complexes behind the implantation region are created (see Fig. 2). As a result a buried noncontinuous $\mathrm{SiO}_{2}$ layer with inclusions of silicon crystallites (10 to $100 \mathrm{~nm}$ dimensions) is formed. It is confirmed by the results of Raman scattering (not shown) and XDS.

In the case of additional carbon implantation, the $\mathrm{SiO}_{2}$ phase appears in the region of carbon implantation, and profile of oxygen distribution shifts to the surface. It is related with two factors, namely: in the region of carbon distribution the critical radius of $\mathrm{SiO}_{2}$ precipitates is noticeably less, which creates conditions for effective rise of the $\mathrm{SiO}_{2}$ phase; drains for interstitial atoms are present, which decrease the precipitation velocity in the region of initial oxygen distribution.

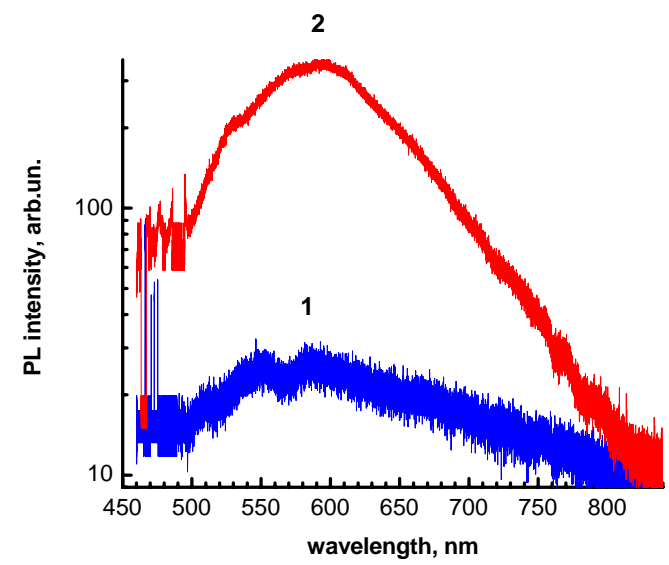

Fig. 5. PL spectra of annealed samples implanted with oxygen (1) and co-implanted with carbon (2). 
Character of the diffuse scattering distribution for the $q_{x}$-curves shows that the displacement fields are close to the spherical ones. For the samples after annealing, a continuous spectrum of small-size defects (from nanometers up to few tens of nanometers) is observed.

PL spectra for the oxygen implanted and carbon coimplanted samples are shown in Fig. 5. After oxygen implantation, the PL intensity within the range 400 to $800 \mathrm{~nm}$ is rather small. For the carbon co-implanted samples, one can observe the intense PL band with a maximum at $600 \mathrm{~nm}$. The shape of this band is complex and may be approximated by a few Gauss curves. In our opinion, PL is caused by silicon nano-crystalline inclusions of 2.2 to $3.0 \mathrm{~nm}$ dimensions in the thin stoichiometric $\mathrm{SiO}_{2}$ layer, which is formed in the samples implanted by oxygen and carbon. The presence of such inclusions is confirmed by our XDS results. Up to date, we are implementing the detail TEM crosssection experiments for a direct confirmation of the nano-inclusion presence.

\section{Conclusions}

It is shown that additional implantation of carbon ions in silicon structures implanted with oxygen leads to redistribution of oxygen along the depth and stimulates $\mathrm{SiO}_{2}$ phase creation in the region between the maximum carbon concentration and maximum concentration of vacancies. A thin $(\sim 30 \mathrm{~nm})$ stoichiometric silicon dioxide layer is formed in this region after annealing. Carbon implantation influences on the defect formation both for initial and annealed samples. For non-annealed samples, carbon implantation leads to decrease of the vacancy-type defect concentration. After annealing of the carbon co-implanted samples, the interstitial defect concentration increases. For these samples, the intense
PL with the peak at $600 \mathrm{~nm}$ is observed, which is associated by us with silicon nano-inclusions in the thin stoichiometric $\mathrm{SiO}_{2}$ layer.

\section{Acknowledgements}

Financial support by the Ministry of Education and Science of Ukraine (Grant M/90-2010) is gratefully acknowledged. Many thanks for the long and very useful scientific discussion to Dr. B. Romanyuk. We also acknowledge the ion implantation group members, in particular G. Kalistyi and V. Fedulov.

\section{References}

1. Y. Wang, R. Smirani, G.G. Ross // Nanotechnology, 15, p. 1554 (2004).

2. A. Romanyuk, V. Melnik, Ya. Olikh, J. Biskupek, U. Kaiser, M. Feneberg, K. Thonke, P. Oelhafen // J. Luminescence, 130, p. 87 (2010).

3. D.I. Tetelbaum, A.N. Mikhaylov, V.K. Vasiliev A.I. Belov, A.I. Kovalev, D.L. Wainstein, Yu.A. Mendeleva, T.G. Finstad, S. Foss, Y. Golan, A. Osherov // Surface \& Coatings Technology, 203 (17-18), p. 2658 (2009).

4. A. Sarikov, V. Litovchenko, I. Lisovskyy, I. Maidanchuk, S. Zlobin // Appl. Phys. Lett. 91, 133109 (2007).

5. V.G. Litovchenko, A.A. Efremov, B.N. Romanyuk // J. Electrochem. Soc., 145, p. 2964 (1998).

6. A. Nejim, Y. Li, C.D. Marsh, P.L.F. Hemment, R.J. Chater, J.A. Kilner, and G.R. Booker // Nuclear Instr. \& Methods in Physics Res., B80/81, p. 822 (1993).

7. V.V. Artamonov, M.Ya. Valakh, N.I. Klyui, V.P. Melnik, B.N. Romanyuk, V.G. Popov, V.A. Yukhimchuk // Functional Materials, 5, p. 551 (1998). 